\title{
Langues et cultures régionales, langues vivantes étrangères Quels enjeux pour une convergence didactique?
}

James Costa

\section{(2) OpenEdition}

Journals

Édition électronique

URL : http://journals.openedition.org/rdlc/6329

DOI : $10.4000 /$ rdlc. 6329

ISSN : 1958-5772

Éditeur

ACEDLE

Référence électronique

James Costa, « Langues et cultures régionales, langues vivantes étrangères Quels enjeux pour une convergence didactique? », Recherches en didactique des langues et des cultures [En ligne], 5 | 2008, mis en ligne le 01 janvier 2008, consulté le 06 novembre 2019. URL : http://journals.openedition.org/ rdlc/6329; DOI : 10.4000/rdlc.6329

\section{(c) $(1) \odot \Theta$}

Recherches en didactique des langues et des cultures is licensed under a Creative Commons AttributionNonCommercial-NoDerivatives 4.0 International License 


\title{
Langues et cultures régionales, langues vivantes étrangères
}

\section{Quels enjeux pour une convergence didactique?}

\section{James Costa}

Institut National de Recherche Pédagogique \& Icar (UMR CNRS 5191), université de Lyon, France

\section{Résumé}

Un établissement scolaire est, en termes écolinguistiques, une écologie à l'intérieur d'une écologie plus large. Les langues que les membres de la communauté scolaire parlent ne sont pas nécessairement celles qui y sont enseignées; des langues de statuts divers s'y côtoient au quotidien.

Certains établissements se trouvent dans une situation particulière du fait de leur localisation dans une région où l'on pratique une langue minoritaire régionale : lorsque cette langue est enseignée, elle fait partie de l'écologie linguistique de l'établissement, qu'elle soit ou non langue maternelle parmi les élèves. De ce fait, et du fait de son statut minorisé dans la société, la langue régionale est souvent exclue du champ de la convergence. Nous essayons de montrer que les enseignants de langues étrangères et de français se privent peut-être d'un auxiliaire précieux.

\section{Mots-clés}

convergence didactique, langues étrangères, langues régionales, écolinguistique

\begin{abstract}
In ecolinguistic terms, a school, whether primary or secondary, can be defined as an ecology within a larger ecology. Some languages are spoken, others are taught, some are both spoken and taught. Languages with various social statuses (or rather their speakers and teachers) live side by side on a daily basis.
\end{abstract}

Yet some schools add a specific dimension to their ecolinguistic setting, i.e. schools that are located in areas where a minority language is or used to be spoken. Once taught, the minority 
language becomes part of the ecology, even though none of the pupils who chose it as a subject might have it as first language. Most other language teachers in France usually choose to ignore that fact, and little collaboration actually takes place between teachers of minority languages and teachers of foreign languages. We would like to suggest that both teachers of foreign languages and teachers of French are thereby depriving themselves of potentially valuable resources.

\section{Keywords}

didactic convergence, foreign language teaching, minority language teaching, ecolinguistics

\section{Introduction}

Comme le rappellent A. Creese et P. Martin (2003),

Despite the increasing amount of literature on the ecology of language, and the link with language policy and planning, there are few studies which focus on the inter-relationships between languages and their speakers in the educational context, specifically, the multilingual classroom.

Si le volume de l'International Journal of Bilingual Education and Bilingualism qu'ils ont dirigé en 2003 permet de commencer à pallier ce manque pour la classe de langue, il n'existe pas à notre connaissance de travaux se penchant sur le contexte écolinguistique des établissements scolaires.

Les concepts développés autour de la notion d'écolinguistique nous paraissent particulièrement adaptés en ce qu'ils permettent d'appréhender la réalité linguistique d'un établissement scolaire dans son intégralité, en intégrant à la fois les langues parlées par les enseignants et les élèves ainsi que la diversité des langues enseignées. Par diversité nous entendons diversité en termes de nombre de langues mais aussi en termes de statut, lorsque l'on considère par exemple un établissement où l'on enseigne une langue régionale ou minoritaire par exemple, ou lorsqu'on englobe les langues de l'immigration.

Nous traiterons ici des questions de convergence didactique, et particulièrement de convergence(s) entre langues régionales de France et langues vivantes étrangères, convergence étudiée ici sous l'angle de l'écologie linguistique. En effet, si les langues régionales s'inspirent des méthodes en vigueur pour les langues étrangères et aspirent ainsi à 
un degré de reconnaissance équivalent, l'enseignement de ces dernières n'a-t-il pas à gagner d'une convergence ou de rapprochements didactiques avec les langues régionales?

Il s'agit avant tout ici d'adapter un cadre théorique particulier à la didactique des langues pour penser la convergence et de proposer des pistes de réflexion. Un autre article serait nécessaire pour traiter la question des liens culturels entre langues, nous ne traiterons ici que des aspects linguistiques.

\section{L'écologie linguistique et les établissements scolaires}

L'idée de convergence didactique répond avant tout au souci de palier l'écartèlement potentiel dont pourraient souffrir les élèves entre les différentes disciplines enseignées en particulier dans le secondaire. Sans lien formel entre elles, elles fonctionneraient chacune en autonomie par rapport aux autres et n'auraient de comptes à rendre à personne en termes de didactique et de concepts employés.

Si une convergence de fait existe par exemple entre les enseignements d'histoire et de géographie (enseignements distincts dans la plupart des autres pays européens), et si une convergence, c'est-à-dire une prise en compte réciproque en termes de reconnaissance et de pratiques pédagogiques, peut exister entre enseignements du français et du latin, voire entre lettres et philosophie, il semble qu'il en aille tout autrement en ce qui concerne l'enseignement des langues. Même si tous, enseignants comme la majorité des élèves, parlent français, il est souvent d'usage d'essayer de réduire le plus possible la part de la langue maternelle au profit de la langue cible dans le cours de langue étrangère. Peu d'enseignants de langue maîtrisent suffisamment une langue étrangère autre que celle qu'ils enseignent pour effectuer des parallèles en cours, ce qui a pour résultat de créer une situation où les langues sont enseignées, en général, de manière hermétique les unes aux autres alors que des stratégies plurilingues pourraient aisément être mises en place. Celles-ci existent de fait, on citera pour mémoire les travaux sur l'éveil aux langues (cf. McPake et Tinsley, 2007) et leur projet Valeur dans le cadre du second projet à moyen terme du Centre Européen des Langues Vivantes (CELV) de Graz qui recense les textes fondateurs européens en la discipline, ainsi que pour une bibliographie exhaustive, ou Candelier (2003) pour une évaluation du programme Evlang) ainsi que les travaux d'intercompréhension entre langues proches (dont les applications pratiques peuvent prendre la forme des Itinéraires romans proposés en ligne par l'Union Latine). 


\section{1. Écologie des langues et écologie linguistique}

La notion d'écologie linguistique nous paraît proposer un point de vue particulièrement intéressant pour traiter de ces questions. Elle apparaît d'abord sous la forme d'écologie des langues chez Einar Haugen (1972) qui définit ce nouveau concept comme

the study of interactions between any given language and its environment. [...] The true environment of a language is the society that uses it as one of its codes. [...] The ecology of language is determined primarily by the people who learn it, use it and transmit it to others.

Haugen (1974: 335) fait remarquer que

the concept of language as a rigid monolithic structure is false. Even if it has proved to be a useful fiction in the development of linguistics, it is the kind of simplification that is necessary at a certain stage of a science but which can now be replaced by more sophisticated models.

Or l'enseignement des langues dans les systèmes scolaires européens en général est l'héritier d'une conception structuraliste de la langue qui en fait un bloc séparé des autres, et cette conception contribue encore aujourd'hui à isoler les disciplines linguistiques les unes des autres, chacun des enseignements répondant par ailleurs à un certain nombre de stéréotypes propres: l'espagnol est une langue de culture (par conséquent on enseigne beaucoup de littérature, d'histoire de l'art en Espagne), l'allemand est une langue très grammaticale (donc très logique), l'anglais est la langue des affaires et elle est simple, elle a peu de grammaire, les autres langues sont marginalisées, et les langues dites régionales, dans ce système établi, sont vues comme étant sans pertinence particulière, survivances d'un état de société ancien que l'on enseigne au titre de la culture locale aux élèves volontaires. Les éventuelles langues maternelles des immigrés sont quant à elles reléguées dans la sphère privée, dans les contextes que nous avons pu observer.

Calvet (1999) propose quant à lui une écologie des langues du monde qui permet de rendre compte des différences de statut entre les langues et de les organiser en un modèle gravitationnel, qui permet selon Sauzet (2008) "d'analisa[r] doncas clarament la tendéncia a l'unilinguisme d'un sistema de comunicacion mondializat".

\footnotetext{
1 "Analyser donc clairement la tendance à l'unilinguisme d'un système de communication mondialisé".
} 
Pour Calvet (1999 : 35),

dans un écosystème linguistique, les langues en présence entretiennent des rapports qui déterminent pour chacune d'entre elles une niche écologique : la 'niche' d'une langue est constituée par ses relations avec les autres langues, par la place qu'elle occupe dans l'écosystème, c'est-à-dire par ses fonctions, et par ses rapports avec le milieu [...].

La notion de milieu est en effet essentielle dans toute approche écologique, on pourrait ainsi qualifier l'établissement scolaire d'écosystème (terme que nous n'adopterons néanmoins pas pour des raisons expliquées plus loin) qui serait constitué de l'ensemble des langues qui y sont en usage, qu'elles soient enseignées ou parlées, ou les deux.

\subsection{De l'écologie des langues aux écologies linguistiques}

On peut ainsi esquisser pour un collège ou un lycée français actuel le schéma gravitationnel suivant (figure 1), qui représente l'écologie des langues dans le cas qui nous occupe - nous ne mentionnons ici que celles qui sont enseignées, les autres n'ayant de fait aucun statut, et leur pratique demeurant largement du ressort du domaine privé :

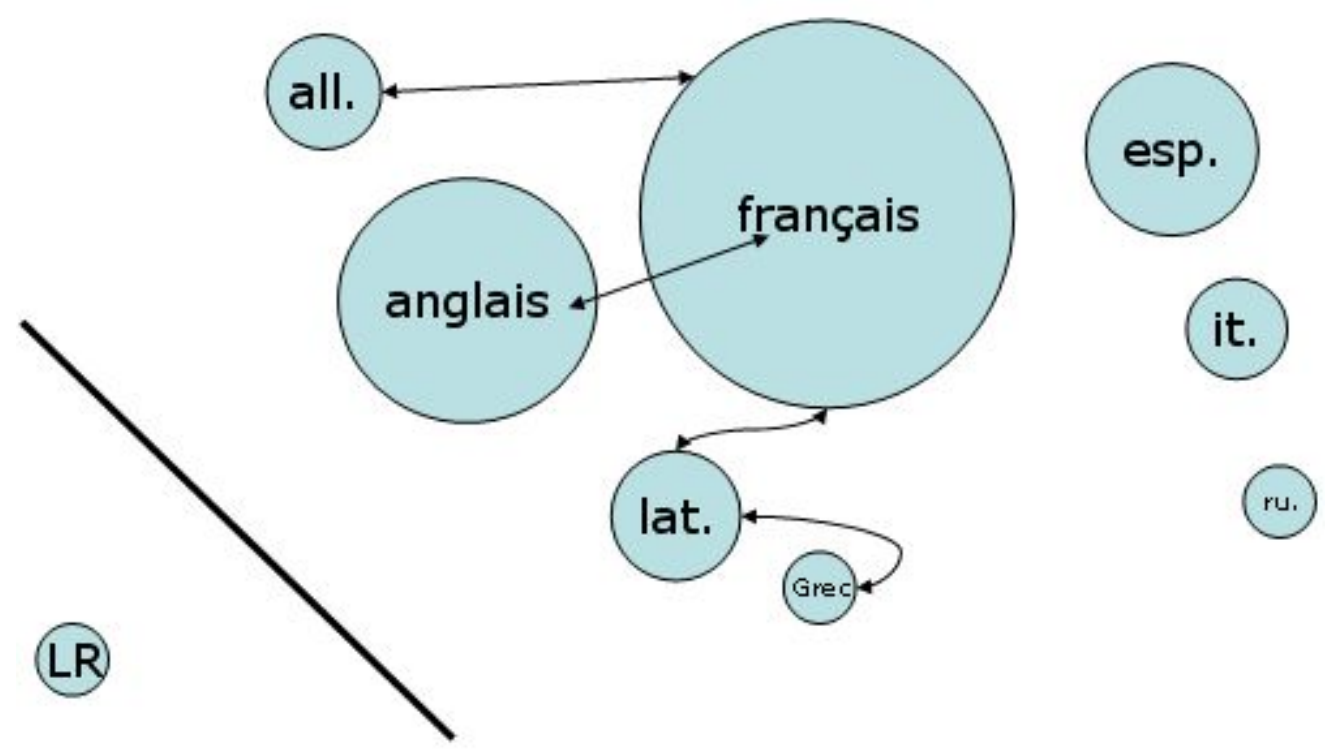

Figure 1 : schéma gravitationnel des langues enseignées dans le secondaire en France aujourd'hui (lat = latin, esp. = espagnol, it. = italien, ru. = russe, all. = allemand, LR = langues régionales).

Dans le schéma, les cercles représentent les principales langues enseignées dans le système scolaire français, avec les liens qui peuvent les unir théoriquement. La taille des cercles 
représente de manière symbolique leur poids au sein de l'institution. La ligne noire symbolise la place à part des langues régionales dans l'Éducation nationale.

Cette représentation pose d'emblée un certain nombre de questions: le français est omniprésent, c'est la langue officielle de la communauté scolaire, pourtant la discipline chargée d'étudier cette langue est considérée au moins autant, sinon plus, comme un enseignement littéraire que comme un enseignement linguistique.

Il y a par ailleurs un certain nombre d'alliances de fait entre certaines langues, alors que d'autres sont mises en concurrence institutionnelle. Du côté des alliances, on peut citer l'alliance d'intérêt entre le français et l'anglais, les deux langues obligatoires (de fait pour l'anglais, comme LV1 ou LV2). L'alliance français / allemand quant à elle est réalisée du côté institutionnel, les autorités éducatives assurant une promotion de l'allemand pour des raisons politiques remontant à l'après-guerre. Il ne s'agit donc pas d'une alliance objective à l'intérieur des établissements mais d'une alliance entre les autorités françaises et allemandes, qui se répercute dans les établissements. Enfin, le latin est un allié traditionnel du français, du fait notamment que les enseignants de latin sont eux-mêmes enseignants de français, et ont intérêt à promouvoir leur discipline pour des raisons à la fois linguistiques et de principe quant à l'enseignement des langues anciennes en France. Du côté de la concurrence, elle se fait d'une part entre espagnol et allemand, pour la LV2, et d'autre part entre l'italien et d'autres langues dites 'rares' par l'Éducation nationale (russe, arabe), entre l'italien et l'espagnol dans les collèges pour le recrutement en LV2. Enfin, les langues régionales, lorsqu'elles sont enseignées, occupent une place à part, soupçonnées ou d'élitisme ou d'inutilité, elles entrent parfois en concurrence avec la LV3, et sont maintenues dans une position défavorable par leur situation institutionnelle et sociolinguistique sur laquelle nous reviendrons plus tard.

La position des langues dans les établissements scolaires entre donc a priori dans une logique de part de marché, logique qui pousse les petites langues (en termes de place dans le système scolaire) à entrer en concurrence entre elles pour se partager ce qu'il reste du gâteau. Cette logique reproduirait, selon Kibbee (2003: 47) les stratégies mises en place par les organismes de promotion de l'anglais (British Council) et du français (Haut Conseil de la Francophonie), logique qui découlerait d'une analyse en termes de marché. 
L'autre logique, concurrente, selon Kibbee serait la vision écologique, représentée par les linguistes travaillant sur la mort des langues, la Linguistic Society of America et MERCATOR en Europe.

Le modèle écologique, s'il peut être contesté par ailleurs, nous semble intéressant en ce sens qu'a priori, le modèle des parts de marché n'est pas le modèle en vigueur à l'école publique.

Si Haugen (1972) parlait d'écologie de la langue (ecology of language), Mühlhäusler (1992) lui préfère la notion d'écologie linguistique, en ce sens qu'elle permet d'appréhender un écosystème dans son ensemble et non plus simplement la position d'une langue particulière. Le terme 'écologie' est préférée à la notion de système (et donc d'écosystème) ${ }^{2}$ :

I have found that the ecological metaphor is particularly productive and a great deal more appealing than a systems metaphor. The latter suggests that we can hope for mechanical, albeit complex explanations or that it is indeed legitimate to study a self-contained system or language as part of a larger system. An ecological view, on the other hand, suggests that we can at best achieve partial and local explanations but that we can hope for understanding and empathy. (1996a : 2)

C'est également le terme que nous retiendrons ici, en parlant de l'écologie d'un établissement scolaire. Pour définir son concept d'ecology (écologie) Mühlhäusler explique (1996b : 205206) que

[t] he notion of ecology suggests both that there are a number of coinhabitants (in our case other languages) and, more importantly, that there are structured relationships between these co-inhabitants and between individual inhabitants and components of the ecological support system.

The key property of any ecology is structured diversity, i.e. diversity defined not so much in terms of numbers but in terms of the quality of meaningful interrelationships. A plural society with many species of different languages that do not interact is not a proper language ecology.

\footnotetext{
${ }^{2}$ La notion d'écologie linguistique' pour désigner non pas la discipline, mais l'objet d'études (l'établissement scolaire est ici vu comme une écologie linguistique) est directement empruntée à Mühlhäusler. La notion d'écosystème implique une relation trop systémique entre les participants de l'écologie, d'où l'adoption ici de ce terme, imparfait en anglais comme en français.
} 
On pourrait alors considérer qu'un établissement scolaire n'est pas une écologie, en tout cas en ce qui concerne toute la partie des enseignements, compte tenu de l'absence de liens qui peuvent exister entre les enseignements de langue. Les interactions informelles seraient le seul moment où l'écologie pourrait fonctionner, hors des cours de langues proprement dits.

Pourtant, nous pensons qu'il serait abusif de ne pas considérer les enseignements de langue comme faisant partie de l'écologie linguistique d'un établissement scolaire, et Haugen (1972) place bien ceux qui enseignent et apprennent les langues au centre de l'écologie des langues. En fait, nous avançons l'hypothèse que les écologies linguistiques qu'ils constituent sont des écologies malades, dans lesquelles dominent deux langues, le français et l'anglais, au détriment de toutes les autres. Leur position hégémonique leur assure un statut tel que les enseignants de ces disciplines n'ont aucun intérêt (en termes de gains de publics ou de prestige) à les ouvrir vers les (autres) langues étrangères, qui ne pèsent rien ou presque, encore moins vers les langues régionales, qui pèsent encore moins mais qui, pour des raisons propres à l'histoire linguistique de France sont encore vues comme autant de menaces potentielles.

Le déficit de communication entre enseignants de langues ainsi que l'absence de travail sur les aspects communs aux différentes langues est patent, ainsi que nous avons pu l'observer dans divers contextes au sein de l'équipe École et Plurilinguisme de l'Institut National de Recherche Pédagogique, et ce dans le primaire comme dans le secondaire, dans les écoles à vocation internationale, dans les collèges et lycées bilingues français / langue régionale et $a$ fortiori dans les établissements scolaires classiques.

Il est par ailleurs intéressant de remarquer que l'écologie linguistique a tendance à être développée pour des situations de revitalisation linguistique et de politique linguistique (Mühlhäusler, 2000 \& 1996a : 2 : "The ecological metaphor in my view is action oriented"). Il s'agit bien, dans le cas d'une écologie déséquilibrée, de gérer l'environnement linguistique en vue d'un retour à une situation considérée comme normale. L'établissement scolaire est un lieu qui se gère, on peut imaginer que les équilibres linguistiques y fassent l'objet de mesures de gestion également.

\section{Les langues régionales dans le secondaire en France et leurs contextes}

Ces considérations initiales sont à notre sens essentielles pour comprendre les enjeux d'une collaboration des enseignants de langue dans un établissement scolaire, ainsi que les enjeux 
de la prise en compte de toutes les langues de la communauté scolaire. Il nous semble artificiel de séparer de manière étanche les cours d'anglais, de français, d'espagnol etc. et dommageable de ne pas prendre en compte les bilinguismes potentiels de certains élèves, ainsi que les répertoires langagiers divers de tous en vue à la fois d'une meilleure compréhension des phénomènes langagiers de la part des élèves (et des enseignants) mais aussi d'une meilleure efficacité des enseignements en question.

Parmi les langues présentes dans les écologies que constituent les établissements scolaires, les langues dites régionales ont une place à part, et ce pour plusieurs raisons, sociales et institutionnelles.

\subsection{Aspects sociaux}

Socialement, les langues régionales de France sont confinées au foyer, elles ne bénéficient d'aucune protection légale, elles n'ont pas droit de cité dans la vie publique et ne font l'objet d'aucune mesure particulière de protection. La Constitution française prévoit depuis 1992 (loi constitutionnelle $\mathrm{n}^{\circ} 92-554$ du 25 juin 1992, cf. Sauzet, 2008) que "[L]a langue de la république est le français". Leur pratique décline partout, et des estimations Insee-Ined faisaient état en 1999 (Héran, Filhon \& Deprez) de 526000 locuteurs pour l'occitan, 304000 pour le breton, 44000 pour le basque, 30000 pour le catalan, 122000 pour le corse. Ces langues évoluent par ailleurs dans des contextes sociolinguistiques très dissemblables : il est difficile de comparer d'une part le breton et l'occitan, langues (presque) exclusivement parlées sur le territoire français, avec le basque et le catalan, qui bénéficient d'une réserve de locuteurs et de rapports de force et de pouvoir bien plus favorables dans l'état espagnol, et sur lesquels les basques et les catalans du nord peuvent s'appuyer. L'alsacien constitue également un cas à part, puisque c'est sous sa forme standard (l'allemand standard) qu'il est enseigné. La transmission familiale des langues autochtones de France est faible dans tous les cas, et les locuteurs âgés.

Pourtant, ces langues sont encore présentes. Ni langues maternelles pour les élèves (moins de 1\% des élèves de 15 ans en France déclarent parler une autre langue non officielle de France chez eux contre 14\% en Espagne [Eurydice, 2005]), ni langues étrangères, ni langues secondes, ni langues de prestige comme pourrait l'être le latin, ni langues permettant d'accéder à un emploi comme l'irlandais, elles sont dans une situation ambiguë. Garabato (2006 : 274) cite ainsi la préface livre de l'élève qui accompagne le manuel d'occitan première année $O c$-ben, paru en 2003, qui résume cette situation : 
[L']originalité de l'enseignement de l'occitan, par rapport à celui des langues étrangères, vient de ce qu'il s'appuie sur un socle d'habitudes culturelles, de pratiques langagières, de savoirs scientifiques que l'élève peut trouver autour de lui ou dont il est lui-même porteur, même s'il n'en a pas toujours conscience.

Nous reviendrons plus loin sur ce statut particulier qui, s'il constitue un handicap pour les langues en tant que telles, pourrait être un atout pour les langues en tant que disciplines scolaires, de par leur situation intermédiaire entre les autres langues enseignées.

\subsection{Aspects institutionnels}

Institutionnellement, certaines langues bénéficient d'un enseignement scolaire (primaire et secondaire) et d'un Capes (occitan-langue d'oc, breton, basque, catalan, corse pour les langues métropolitaines, le gallo étant enseigné comme option facultative évaluée au baccalauréat en Haute Bretagne sans faire l'objet d'un Capes).

$\mathrm{La}$ situation scolaire des langues régionales est particulière. Scolairement, elles existent, et ce depuis la Loi Deixonne de 1951, abrogée depuis ${ }^{3}$. Pourtant, elles ne font partie du socle commun sous aucune forme, et les Capes qui certifient les enseignants de langues régionales dans le secondaire sont bivalents (le corse mis à part), c'est-à-dire que les enseignants sont certifiés en langue régionale mais aussi dans une autre discipline, le plus souvent l'histoiregéographie ou le français, voire l'anglais. Ce n'est pas le principe en soi qui est à mettre en cause, mais il a pour conséquence que de nombreux enseignants n'ont pas de formation de linguistes et viennent de filières telles que les lettres ou l'histoire.

La discipline 'langue régionale' à l'école est appelée Langue et Culture Régionale (LCR), alors que les langues étrangères sont appelées Langues Vivantes Étrangères (LVE), ce qui induit immédiatement des représentations différences. Le français quant à lui a un statut à part puisqu'on l'appelle simplement 'français'. Les langues régionales, ainsi que le français, sont pourtant également des langues vivantes.

Les cours de LCR sont placés à des horaires prompts à décourager de nombreux élèves, avec des cours souvent enseignés pendant la pause déjeuner des autres élèves, de manière à regrouper des élèves de classes différentes. Lors de l'une de nos enquêtes en Bretagne, les élèves se sont également plaints que la salle de breton, étant placée en bout de couloir, ne

\footnotetext{
${ }^{3}$ Pour un historique des lois scolaires sur les langues de France, voir Garabato (2006).
} 
recevait pas le chauffage : la salle était froide tout l'hiver. Dans les rectorats, les LCR sont gérées par la personne s’occupant des langues rares.

Enfin, l'organisation des cours de LCR peut se faire suivant des modalités variables selon les lieux, les volontés locales, la force des militants locaux ou des parents, la volonté du chef d'établissement et des autorités académiques. Ces enseignements vont de l'option facultative (de une à trois heures hebdomadaires) à partir de la sixième jusqu'au baccalauréat, à la possibilité de choisir la LCR comme LV2 ou LV3, possibilité rarement donnée qui a l'inconvénient de mettre la langue régionale en concurrence directe avec les LVE. Une possibilité supplémentaire existe qui permet la création de filières bilingues, depuis la maternelle jusqu'à la terminale (dans le secondaire, en fait, il s'agit le plus souvent d'un enseignement renforcé de LCR et de l'enseignement d'une discipline, le plus souvent l'histoire-géographie, en langue régionale). Ces filières touchent relativement peu d'élèves : environ 5000 pour le corse, 5500 pour l'occitan, 10000 pour le breton (source Flarep), mais elles ont le mérite d'exister, et elles se développent. Au total, ce sont plus de 350000 élèves qui, en 2003-2004, étaient concernés par un enseignement de langue régionale en France, toutes langues confondues $\square$ y compris les langues des Départements et Territoires d'OutreMer $\square$ (Éduscol, 2005), soit une proportion infime.

En termes d'objectifs, l'Éducation nationale fixe pour les LVE des critères précis d'évaluation et d'objectifs (circulaire n²006-093 du 31-5-2006 Dgesco) :

L'article ler du décret définit les objectifs visés aux différentes étapes du cursus scolaire en langues:

- utilisateur élémentaire niveau A1 (introductif ou découverte) pour la fin des études primaires;

- utilisateur indépendant niveau B1 (seuil) pour la fin de la scolarité obligatoire;

- utilisateur indépendant niveau B2 (avancé ou indépendant) pour la fin des études secondaires.

Le niveau A2 (utilisateur élémentaire intermédiaire ou usuel) constituera la référence pour le socle commun.

Ces critères deviennent nettement plus flous en ce qui concerne les LCR : "L'Éducation nationale se doit de faire vivre ce patrimoine culturel, de veiller au développement des langues régionales et de contribuer à leur transmission" (Encart B.O. n³3 du 13-09-2001) 
et, concernant l'enseignement bilingue, il s'agit "d'atteindre un niveau de communication et d'expression écrite plus performant" tout en "confort[ant] l'apprentissage du français et [en] prépar[ant] les élèves à l'apprentissage d'autres langues" (Boen du 13/09/2001).

Les programmes scolaires assignent aux langues régionales des objectifs vagues et non déterminés avec autant de précision que pour les LVE, même si l'idée de transmission est présente et qu'elle permet de nombreuses interprétations plus ou moins favorables. Pourtant, on comprend qu'il s'agit de transmettre des savoirs, un patrimoine, mais il n'est pas question de compétences linguistiques et sociolinguistiques.

À ces éléments sociétaux et institutionnels qui tendent à marginaliser la place des LCR dans l'écologie linguistique des établissements scolaires, on pourrait rajouter le fait que celles-ci sont souvent vues négativement par les autres disciplines, pour diverses raisons (sociolinguistiques, mais aussi concurrence au niveau des LV3, temps qui pourrait être consacré à autre chose). Une analyse succincte des manuels de lettres de $5^{\mathrm{e}}$ (année où est abordée la question de la langue médiévale et de l'existence de la langue d'oc au moyen âge) ou de géographie de $4^{\mathrm{e}}$ (moment du programme dans lequel on aborde la question des langues en Europe) permet de voir que la question des minorités linguistiques est soit ignorée, soit abordée sous l'angle du conflit. Lorsqu'une carte des langues d'Europe est présentée, elle est presque systématiquement fausse (ainsi la langue de la Lettonie serait le lettonien (sic) selon Champigny, 2002).

Ainsi, à propos des langues, on peut lire par exemple (Champigny, 2002) :

Au fil des guerres qui ont déchiré l'Europe, les frontières des États se sont déplacées et surtout multipliées. Elles ne correspondent pas toujours aux limites des langues ou des religions, ce qui est source de tensions.

Ou encore, dans le même manuel, à propos des territoires périphériques de la France (ici le Midi) :

En France, regarder vers Toulouse, c'est regarder vers des lieux où le soleil est plus haut dans le ciel à "midi ». De là, ces régions du Sud tirent leur nom et leur image de marque. Nous pourrions y ajouter la mer, les cigales, les oliviers, l'odeur du maquis, de la garrigue et des pins! - Il faudrait aussi pour faire le tour de cette vision traditionnelle du midi parler de la culture de la vigne, des fruits et des agrumes rendue possible par l'irrigation qui permet de combattre la sécheresse. Il faudrait enfin évoquer 
le grand développement du tourisme. Pourtant le midi ne se réduit pas à des clichés, et, surtout, n'est pas uniforme. (Champigny, 2002)

Nous avons présenté ici le contexte sociétal et institutionnel (scolaire) qui peut expliquer au moins en partie pourquoi les langues régionales bénéficient d'une reconnaissance si minime au sein des établissements scolaires et ont une place si infime dans leurs écologies linguistiques respectives. Les convergences et les travaux en commun entre disciplines sont loin d'aller de soi (hors Itinéraires de découvertes en collège et Travaux personnels Encadrés en lycée) lorsque l'on parle des disciplines prestigieuses (français et anglais pour les disciplines à contenu linguistique). On comprend qu'elles soient encore moins pensables dans le cas des langues régionales.

\section{Une impossible convergence?}

Les écologies linguistiques sont des milieux complexes, et certaines langues jouent à travers leur présence institutionnelle à l'école une grande partie de ce que sera leur futur, si elles en ont un. Par là, nous entendons que la présence à l'école est vue par les militants des langues régionales comme une manière sinon d'arrêter du moins de ralentir le mouvement de substitution linguistique qui est en cours d'achèvement en France. Pour ces langues, l'identification aux langues vivantes étrangères est un objectif lointain en termes de statut, statut qui est perçu comme enviable de par l'officialité institutionnelle dont elles bénéficient, sachant qu'il est peu probable que les langues régionales acquièrent un statut similaire à celui $\mathrm{du}$ français dans un avenir proche.

\subsection{Quand les langues régionales regardent vers les langues vivantes}

Si les enseignants de LVE ignorent les langues régionales dans les pratiques pédagogiques observées (ce qui ne les empêche pas d'être par ailleurs locuteurs), les langues étrangères constituent le modèle des enseignants de langues régionales, et compte tenu du contexte c'est plus que normal. En termes de pédagogie, la réflexion sur les langues étrangères est naturellement plus avancée, compte tenu des moyens financiers investis dans les recherches pour la didactique de l'anglais par exemple, en France mais surtout en Grande-Bretagne et aux Etats-Unis.

Les ressources disponibles en langues régionales augmentent chaque année, mais elles sont comparativement peu nombreuses par rapport aux langues étrangères, et les LVE définissent 
toujours la norme en discipline d'enseignement des langues, pour les élèves comme pour les enseignants. Ainsi, le manuel d'occitan Oc-ben est basé sur un livre de portugais, mais des limites sont vite atteintes, du fait même de l'environnement limité de la langue occitane (Garabato, 2006) :

[la méthode] la plus novatrice est probablement Oc-Ben! qui en apparence n'est pas différente d'une méthode de FLE. L'occitan est 'clairement abordé comme une langue de communication' d'après les auteurs, qui s'adressent aux jeunes. [...] [L]e contenu du livre montre bien la réalité sociolinguistique de l'occitan: textes littéraires, chansons, dialogues inventés et images fabriquées qui 'occitanisent' le quotidien pourtant toujours en français [...] en définitive et inévitablement un monde de fiction....

De même, lors d'une conversation particulière, un inspecteur d'académie des Bouches-duRhône confiait qu'il était vital pour la crédibilité des langues régionales de se calquer sur les méthodes traditionnelles d'enseignement du français et des langues vivantes. C'est également une attente de la part des élèves, qui attendent du cours d'occitan un cours traditionnel calqué sur le cours d'anglais, comme nous avons pu le constater dans plusieurs collèges de la Drôme.

Les enseignants de langues régionales sont de fait prêts à la convergence, autant pour la convergence elle-même que pour des questions de reconnaissance de leur discipline, et ce sont bien souvent les seuls à être formés aux problématiques liées au bilinguisme et à s'y intéresser activement, voire à encourager les élèves allophones à ne pas avoir honte de leur langue maternelle et à la valoriser (Costa, 2008a). Ainsi, une enseignante d'occitan d'Albi, s'exprimant ici en occitan alpin lors d'un entretien réalisé en mai 2007 par internet, déclare :

James Costa : e tu, fàcia an aqueu bilinguisme, coma reagisses ? MB : bilinguisme amb la lenga d'immigracion ? MB : ben penso que siau la sola professora a aver cercat a saber $M B$ : après d'expleitar en classa es pas evident $\mathbf{M B}$ : mai lo valoriso e lor ai explicat qu'èra un astre per elei ${ }^{4}$.

Le plurilinguisme est non seulement une évidence pour un enseignant de langue régionale, c'est également une manière de valoriser et de promouvoir sa discipline en mettant en avant

\footnotetext{
${ }^{4}$ James Costa : et toi, par rapport à ce bilinguisme, comment réagis-tu? MB : bilinguisme avec la langue d'immigration? MB : ben je pense que je suis la seule enseignante à avoir cherché à savoir MB : après à exploiter en classe ça n'est pas évident MB : mais je le valorise et je leur ai expliqué que c'était une chance pour eux.
} 
une ouverture vers des langues jugées plus prestigieuses, langues romanes pour l'occitan (voir par exemple Agar, 2002), le corse et le catalan, langues celtiques et anglais pour le breton.

La romanité en particulier est très peu prise en compte dans les programmes scolaires, et les diverses langues romanes enseignées obéissent largement à une logique disciplinaire, même si des dispositifs de type 'parcours romans' (intégrant l'occitan) existent ici et là.

Les enseignements de langues régionales permettent aussi une ouverture vers le français par la prise en compte des interlectes régionaux. Nous pensons ici particulièrement au francitan qui est utilisé comme tremplin vers l'occitan ainsi que pour une meilleure maîtrise du français standard par de nombreux enseignants. L'occitan est à Marseille la seule discipline scolaire qui prenne en compte la spécificité locale des répertoires langagiers des élèves et qui les valorise pour rebondir ensuite sur l'occitan marseillais et le français standard.

Enfin, les langues régionales sont les seules langues dans lesquelles est dispensé un véritable enseignement bilingue à parité horaire dans le primaire, le plus souvent (en Catalogne, Bretagne et Provence) dans une logique où un maître enseigne dans les deux langues, gérant ainsi sa classe au mieux et faisant les ponts nécessaires entre les langues.

\subsection{Vers une écologie linguistique restaurée?}

On le voit, l'enseignant de langues régionales est, potentiellement, celui qui peut justement faire lien entre la langue désormais maternelle et les langues étrangères, de même que la langue régionale, de par sa position dans le continuum existant (pour les langues romanes) entre le français régional parlé et les langues étrangères proches, peut servir de lien et de pont entre ces diverses variétés ainsi que de base pour une réflexion métalinguistique.

L'enseignement des langues régionales, les enseignants l'ont bien compris, a tout à gagner d'un rapprochement et d'un travail convergent avec les langues étrangères et le français, en termes d'efficacité comme en termes d'image et de représentations, et rien à gagner d'une logique d'affrontement frontal ou d'isolement, comme le montre Eggington (2001 : 249) :

Rather, presenting a [revitalisation] plan in terms of both power and solidarity can strengthen the possibilities of long-term support and development from both the minority and majority culture - especially majority cultures.

C'est bien de planification linguistique qu'il s'agit là, et d'une planification qui ne peut se permettre de se faire au détriment de l'une ou l'autre des composantes linguistiques (les "co- 
inhabitants" dont parlait Mühlhäusler, 1996b) de l'établissement scolaire. Dans une écologie linguistique saine, "the majority of [...] links are mutually beneficial (90 per cent) and only a small number competitive or exploitative" (Mühlhäusler, 2002 : 38).

Les langues régionales ont besoin, nous l'avons dit, de la convergence avec les langues les plus fortes de l'écologie linguistique qu'est l'établissement scolaire. Il nous semble pourtant qu'à la lumière de ce que nous avons présenté, les enseignants de LVE et de français peuvent également trouver leur intérêt et celui de leur discipline dans une convergence entre leurs disciplines et l'enseignement des langues régionales pour des raisons qui sont multiples :

- tous enseignent en effet un objet 'langue'.

- dans la réalité, les pratiques des élèves, les langues sont imbriquées les unes dans les autres, du fait des continuums entre français, français régional, accents et langue régionale, des histoires familiales, des proximités linguistiques entre langues de même famille linguistique. Les élèves ont tous à leur disposition un certain nombre de répertoires langagiers dont les

positionnements [par les locuteurs eux-mêmes] permettent de mieux comprendre les orientations de l'investissement des locuteurs dans l'apprentissage de certaines langues, pour le capital qu'elles représentent sur le marché des langues, sans être pour autant liées au nombre de locuteurs réels recensés (Moore, 2006 : 79).

- Les langues enseignées sont souvent des langues voisines (français, italien, espagnol, sans oublier l'anglais dont le lexique est à 50\% roman) ou collatérales ${ }^{5}$ (occitan, corse, catalan, langues d'oïl, francoprovençal). On peut aisément faire apparaître des ponts entre les langues, qu'ils soient lexicaux, syntaxiques ou sémantiques. Il s'agit là d'éléments qui peuvent faciliter des processus de convergence didactique. Les instructions officielles en langues régionales demandent par ailleurs que leur enseignement s'ouvre

\footnotetext{
${ }^{5}$ Voir Eloy (2004) pour une définition précise de la notion de collatéralité. Il s'agit de langues qui sont dans un rapport de force défavorable avec une langue dominante qui leur est proche, comme le français et le gallo.
} 
aux communautés linguistiques proches [...]. Cette perspective [...] s'inscrit également dans dans la volonté de faire de cet enseignement un élément de la construction de l'identité européenne (Boen n³3 du 13/09/2001).

La prise en compte non-idéologique de l'ensemble des langues de la communauté scolaire (y compris donc les langues régionales ou de l'immigration lorsqu'elles sont présentes) est le seul moyen de rétablir une écologie linguistique saine. La mise en place d'actions convergentes peut être ainsi vue comme contribuant à une réelle micro-politique linguistique au niveau de l'établissement scolaire pouvant aboutir à un véritable plurilinguisme (c'est-àdire une écologie linguistique européenne saine pour reprendre la terminologie écolinguistique) scolaire comme le préconisent les instances européennes (cf. par exemple Maalouf, 2007).

\section{Conclusions}

Les établissements scolaires, nous l'avons vu, peuvent être représentés, de par la diversité des langues qui s'y côtoient, comme des écologies linguistiques, mais des écologies linguistiques fragiles ou malades. Il y existe une nette hiérarchie des langues, qui conditionne les rapports pédagogiques entre enseignants.

Les contacts inter-langues sont rares, et laissent de côté les langues de l'immigration ou les langues régionales, c'est-à-dire les langues dont le statut est le plus bas. Ces considérations vont à l'encontre d'une conception écolinguistique qui plaide contre les dangers du monoculturalisme et du monolinguisme et pour une prise en compte des facteurs qui réduisent ou au contraire contribuent à améliorer la santé de ces écologies.

La convergence didactique, et le dialogue qu'elle suppose, entre langues ou avec d'autres disciplines non strictement linguistiques nous semble un moyen efficace d'y contribuer. Les enseignants de langues régionales, pour les raisons que nous avons rappelées, nous semblent particulièrement à même de jouer un rôle pivot dans la mise en place d'une politique linguistique commune au sein des établissements, et il nous semble que la mise en place de ce type de mesures illustrerait bien le principe écologique collaboratif dont parle Mühlhäusler (2002), en ce sens que si les enseignants de langues régionales ont besoin de la reconnaissance et de l'appui de leurs collègues de LVE ou de français, ces derniers ont tout autant besoin des enseignants de langues régionales et de leurs savoir-faire, de leurs outils, de leurs approches. Ni langues étrangères, ni langues maternelles, ni langues secondes, les 
langues régionales ne peuvent elles au moins temporairement assumer le rôle de langues liens ou de langues ponts?

\section{Références}

\section{Bibliographie}

Agar, D. (2002). Palancas. Fichièr autocorrectiu d'entraïnament ortografic occitan-francés. Tolosa : La Poesia.

Bastardas-Boada, A. (1996). Ecologia de les llengües. Medi, contactes i dinàmica sociolingüística. Barcelona : Proa.

Calvet, L.-J. (1999). Pour une écologie des langues du monde. Paris : Plon.

Candelier, M. (2003). L'éveil aux langues à l'école primaire : Evlang : bilan d'une innovation européenne. Bruxelles : de Boeck.

Champigny, D. (dir.) (2002). Histoire-Géographie 4e. Paris : Nathan.

Costa, J. (2008). "Immigration, Plurilingualism and Minority Language Teaching in France :

Advantages and Prospects, a Sociodidactic Perspective". Multilingualism, Citizenship, and the Future of Minority Languages. Actes du Colloque de la 11th International Conference on Minority Languages. Pécs. À paraître.

Creese, A. \& Martin, P. (2003). "Multilingual classroom ecologies : inter-relationships, interactions and ideologies". International Journal of Bilingual Education and Bilingualism, vol. $6, \mathrm{n}^{\circ} 3$ \& 4 . pp. 161-167. Disponible en ligne. http://www.multilingualmatters.net/beb/006/beb0060161.htm

Eloy, J.-M. (2004). Des langues collatérales. Problèmes linguistiques, sociolinguistiques et glottopolitiques de la proximité linguistique. Actes du Colloque international réuni à Amiens, du 21 au 24 novembre 2001. Paris : L’Harmattan.

EDUSCOL (2005). "Bilan de l'enquête sur les enseignements des langues et cultures régionales en 2003-2004". Disponible en ligne, consulté en février 2008 : http://eduscol.education.fr/D0072/enqueteLR2005-2.htm 
Eurydice (2005). Chiffres clés de l'enseignement des langues à l'école en Europe. Bruxelles : Commission Européenne.

Eggington, W.G. (2001). "Language revitalisation planning within a power / solidarity framework". Current Issues in Language Planning, vol. 2, n 2 \& 3. pp. 242-250.

Fill, A. \& Mühlhäusler P. (dir.) (2001). The Ecolinguistics Reader. London \& New York : Continuum.

Garabato, C. A. (2006). "Enseigner l'occitan / en occitan aujourd'hui : un parcours du combatant". Boyer, H. (dir.) Études de Linguistique Appliquée, n ${ }^{\circ}$ 143. pp. 265-279.

Haugen, E. (1972). "The ecology of language". In Dil A.S. (dir.). The Ecology of Language : Essays by Einar Haugen. Stanford: Stanford University Press. pp. 325-339.

Héran, F., Filhon, A. \& Deprez, C. (2002). "La dynamique des langues en France au cours du XXe siècle". Populations et Société n ${ }^{\circ}$ 376. pp. 4.

Maalouf A. (2007). Un défi salutaire: comment la multiplicité des langues pourrait consolider l'Europe. Bruxelles : Commission Européenne. Disponible en ligne, consulté en février 2008: http://ec.europa.eu/education/policies/lang/doc/maalouf/report_fr.pdf

McPake, J. \& Tinsley, T. (2007). Valoriser toutes les langues en Europe. Graz: ECML/CELV. Disponible en ligne, consulté en janvier 2008 : http://www.ecml.at/mtp2/publications/Valeur-report-E.pdf

Moore, D. (2006). Plurilinguismes et école. Paris : Didier.

Mühlhäusler, P. (1992). "Preserving languages or language ecologies? A top-down approach to language survival". Oceanic Linguistics, vol. 31, n² . pp. 163-180.

Mühlhäusler, P. (1996a). Linguistic ecology. London \& New York : Routledge.

Mühlhäusler, P. (1996b). "Ecological and non-ecological approaches to language planning". In Hellinger, M. \& Ammon, U. (dir.). Contrastive sociolinguistics. Berlin \& New York: Mouton de Gruyter. 
Mühlhäusler, P. (2000). "Language planning and language ecology". Current Issues in Language Planning, vol. 1, n 3. pp. 306-367.

Mühlhäusler, P. (2002). "Why one cannot preserve languages (but can preserve language ecologies)". In Bradley D. \& Bradley M. (dir.). Language endangerment and language maintenance. London : RoutledgeCurzon.

Sauzet, P. (2008). "Se pòt existir una lenga sensa marina de guèrra ? A prepaus de l'occitan". Llengua, Societat i Comunicació, nº 6 (à paraître). Barcelona : Universitat de Barcelona.

Salles-Loustau, J. (dir.) (2003). Oc-ben. Paris : CNDP / SCERÉN.

\section{Sites Internet}

FLAREP (Fédération pour les Langues Régionales à l'École Publique). Consulté en février $2008: \underline{\text { http://www.flarep.com }}$

Language Ecology Research Forum website. Consulté en février 2008: http://www.ecoling.net/

Parcours romans. Sur le site de l'union latine. Consulté en janvier 2008: http://dpel.unilat.org/DPEL/Creation/IR/index.fr.asp

Socle commun de compétences (2006). Ministère de l'Éducation nationale. Consulté en décembre 2008: http://www.education.gouv.fr/cid2770/le-socle-commun-de-connaissanceset-de-competences.html

\section{À propos de l'auteur}

James Costa est chargé de recherche à l'Institut National de Recherche Pédagogique au sein de l'équipe École et plurilinguisme, rattachée au laboratoire Icar (UMR 5191, Cnrs \& Université de Lyon). Il travaille principalement en sociolinguistique sur les classes bilingues français-langues régionales et sur la problématique des langues en danger.

Courriel : james.costa@ens-1sh.fr.

Adresse : Laboratoire Icar, École Normale Supérieure - Lettres et Sciences Humaines, 15 Parvis René Descartes, 69342 Lyon Cedex 07, France. 